\title{
Étude de la reconnaissance professionnelle des $\mathrm{AESH}^{*}$
}

\author{
Mickaël Jury ${ }^{1}$, Julie Bergara ${ }^{2}$, Grégoire Cochetel ${ }^{1}$ \\ ${ }^{1}$ Université Clermont Auvergne, ACTé \\ ${ }^{2}$ Académie de Bordeaux,
}

\begin{abstract}
Résumé
Les Accompagnant·es d'Élèves en Situation de Handicap (AESH) sont essentiel·les au bon fonctionnement de l'école inclusive mais ressentent régulièrement un manque de reconnaissance de la part de l'institution. Cette étude vise à étudier de manière quantitative cette question en s'intéressant notamment aux paramètres pouvant faire varier cette reconnaissance perçue. Réalisée auprès de $2203 \mathrm{AESH}$, cette recherche a permis de confirmer qu'elles perçoivent moins de reconnaissance de la part de l'institution que de la part du personnel de direction ou des enseignant $e s$ et que cette reconnaissance est fortement tributaire de l'accueil réalisé dans l'école ou l'établissement ou encore des relations de travail avec l'enseignant $e$. Ces premières données, non sans limites, ouvrent des perspectives de réflexion afin de permettre à ce personnel de pleinement contribuer au parcours inclusif des élèves.
\end{abstract}

Mots-clés : Scolarisation inclusive ; Handicap ; Reconnaissance professionnelle ; Accompagnement ; Questionnaire

Les Accompagnantes ${ }^{\dagger}$ d'Élèves en Situation de Handicap (AESH) contribuent à la scolarisation des élèves en situation de handicap. Plus précisément, et pour permettre le développement de l'autonomie, elles ont, d'après la Circulaire n 2017-084 du 3-5-2017, une mission d'aide auprès de ce public concernant les activités du quotidien (par ex., les actes essentiels de la vie), l'accès aux activités d'apprentissages (par ex., contribuer à l'adaptation de la situation d'apprentissage) ou encore les activités de la vie sociale et relationnelle (par ex., favoriser la communication et les interactions entre l'élève et son environnement). Ces actrices constituent donc un rouage essentiel du parcours de scolarisation inclusif d'élèves en situation de handicap et expriment pourtant régulièrement un manque de reconnaissance de la part de l'institution. Dans le cadre de cette recherche, nous souhaitons étudier plus spécifiquement cette question via une étude ayant deux objectifs : la description des conditions de travail des AESH et l'examen des liens entre celles-ci et leur reconnaissance professionnelle perçue. Ainsi, après avoir évoqué le métier d'AESH et la question de la reconnaissance perçue, nous présenterons une étude quantitative exploratoire réalisée auprès d'un nombre conséquent d'AESH.

\footnotetext{
* Ce papier a été accepté pour publication dans La nouvelle revue - Education et société inclusives. II s'agit de la version post-print. Les communications sont à adressées à mickael.jury@uca.fr ${ }^{\dagger} \|$ est à noter que pour des questions de lisibilité le féminin sera employé pour désigner les AESH dans cet article. En effet, que ce soit dans les chiffres officiels (voir MEN, 2019) ou dans cette étude (voir partie Méthode), il s'agit d'un personnel largement féminin et il nous semble ainsi important de le reconnaître. Ce choix n'implique pour autant pas que nous pensions que cette profession soit réservée aux femmes ni que les hommes y sont moins légitimes.
} 


\section{AESH, une Profession Singulière dans l'Éducation Nationale}

Cochetel (2019), dans son ouvrage, retrace l'évolution de cette profession au sein de l'Éducation nationale en soulignant la particularité française associée à celle-ci. Ainsi, des AIS (Auxiliaire d'intégration scolaire) aux AESH en passant par les AVS (Auxiliaire de vie scolaire), il indique que la France a depuis une quarantaine d'années voulu soutenir la participation des élèves en situation de handicap en leur permettant d'être accompagnés par du personnel non enseignant dans les classes. Néanmoins, ces dénominations successives traduisent pour l'auteur la difficulté rencontrée par l'autorité compétente pour définir un métier et ses missions de manière claire.

Il faudra notamment attendre le rapport Komitès (2013, voir aussi Philip \& Philbert, 2009) sur la professionnalisation des accompagnantes pour la réussite des enfants et adolescents en situation de handicap, pour parvenir, via le décret n²014-724 du 27-6-2014, à la création d'un « quasi-statut » pour ce personnel. Toutefois, bien que les améliorations apportées par celui-ci soient sensibles (par ex., possibilité d'être recrutée en $\mathrm{CDI}$ ), il semble que « l'affirmation d'un métier à part entière [doive] attendre un peu » (Cochetel, 2019, p. 16).

En outre, si le cadre de cet emploi semble si difficile à poser, cela s'explique aussi par la pluralité de situations auxquelles ce personnel pouvait faire face. Ainsi, jusqu'aux récentes transformations, il n'était pas rare de trouver pour une même fonction de multiples statuts (par ex., assistant es d'éducation faisant fonction d'AVS, EVS via un contrat aidé, AESH via un contrat de droit public) se traduisant par des cadres et des volumes d'emploi, des temps de travail, ou des référent-es différents (par ex., possibilité de tâche administrative pour les EVS, aide individuelle auprès d'un élève et en même temps aide mutualisée auprès de plusieurs).

Si la récente création des pôles inclusifs d'accompagnement localisés via la Circulaire n²019088 du 5-6-2019 a notamment eu pour objectif de contribuer à la professionnalisation de la fonction d'AESH et faciliter leur gestion des ressources humaines, il n'en demeure pas moins que les quelques éléments mentionnés ici illustrent les difficultés entourant la définition de cette profession et peuvent ainsi contribuer à expliquer pourquoi certaines AESH perçoivent une faible reconnaissance de leur travail.

\section{Reconnaissance Professionnelle Perçue}

La recherche de reconnaissance au sens large constitue une motivation fondamentale de l'être humain (Anderson et al., 2015). Selon ces auteur.rices, voir notamment sa compétence reconnue par autrui est associé au bien-être, à l'estime de soi ainsi qu'à une bonne santé physique. Dans le contexte professionnel, Brun et Dugas (2005, p. 85) ont proposé de définir la reconnaissance comme étant un « jugement posé sur la contribution de la personne, tant en matière de pratique de travail qu'en matière d'investissement personnel et de mobilisation ». Aussi, Fall (2015) indique-t-il que cette reconnaissance peut être matérielle (par ex., via une augmentation de la rémunération) ou immatérielle (par ex., via des compliments sur le travail réalisé) et qu'elle vise à encourager l'émergence de certains comportements au travail. Il est à noter que celle-ci peut varier selon les sources. Ainsi, Brun et Dugas (2005) indiquent que la reconnaissance peut notamment se situer au niveau institutionnel (c.-à-d., dans les moyens et politiques mis en place par l'organisation pour reconnaître le travail d'une cible en particulier), au niveau 
vertical (c.-à-d., la reconnaissance immédiate que la·le supérieur·e hiérarchique accorde à l'employé·e) ou encore au niveau horizontal (c.-à-d., la reconnaissance portée par les collègues ou les pairs).

El-Akremi, Sassi, et Bouzidi (2009) proposent que ce besoin de reconnaissance, notamment dans le contexte professionnel, trouverait son origine dans l'instabilité des identités professionnelles et conditions de travail des salarié ·es (voir aussi Brillet \& Capdevielle, 2017). Ce concept n'est donc pas à négliger puisqu' il apparaît comme étant relativement déterminant dans les situations professionnelles en étant associé à de multiples conséquences comme la motivation intrinsèque à réaliser son travail (c.-à-d., faire son travail pour la satisfaction qu'il procure, Fall, 2015) ou encore l'évitement du burnout (c.-à-d., le syndrome d'épuisement professionnel, Renger et al., 2020).

Dans le cas où une forte inconnue autour d'un contexte de travail amènerait les travailleur euses à rechercher davantage de reconnaissance de la part d'autrui, il n'est alors pas surprenant que les AESH puissent être particulièrement sensibles à cette question et exposées à un risque d'épuisement (Maguet \& Panissal, 2019).

\section{Reconnaissance Professionnelle des AESH}

Comme suggéré auparavant, la question de la reconnaissance des AESH est particulièrement vive sur le terrain. À titre d'illustration, citons ce témoignage d'« une AVS qui aime son travail » (2017), indiquant qu'elle ne peut pas, avec son emploi, « se prévaloir d'une reconnaissance sociale, ni d'utilité publique, ni de valeur, ni de compétence » (p. 62).

Les organisations syndicales ou les collectifs d'AESH sont ainsi extrêmement mobilisés sur cette question. Récemment, une étude réalisée auprès d'AESH par le syndicat national des lycées et collèges (SNALC) en 2017 concluait qu'un bon nombre de ces salariées expriment "un manque de considération tant au niveau de la hiérarchie, du chef d'établissement, de l'inspection, des services du rectorat que de certains enseignants ». Autre exemple plus récent, une déclaration intersyndicale datant de 2019 en réaction à la concertation sur l'école inclusive portée conjointement par le ministère de l'Éducation nationale et le secrétariat chargé des personnes handicapées demandait la reconnaissance professionnelle immédiate des AESH (à travers notamment une revalorisation salariale mais aussi « la création d'un vrai statut pour les AESH »). Toutefois, malgré la tension autour de cette question, peu de travaux s'y sont spécifiquement intéressés.

Des publications, parmi lesquelles celle de Maguet et Panissal (2019,) illustrent, au moins indirectement, certaines préoccupations éprouvées par les AESH et notamment le manque d'intégration au sein des équipes éducatives. Les autrices citent ainsi les propos de trois AESH qui expriment nettement leur inconfort dans certaines situations de leur quotidien professionnel (par ex., non informées des changements d'emploi du temps, se sentent seules dans la réalisation de leurs activités). Dans le même ordre d'idée, Toullec-Théry (2019) suggère que certain ·es enseignant·es, par manque de travail de préparation, peuvent maintenir les AESH à distance et, de fait, créer des situations professionnelles potentiellement difficiles pour ces dernières. Thomazet et Mérini (2015) expliquent que la source de ces difficultés est liée à l'espace de travail commun, trop peu partagé ou co-construit par les différents professionnel-les. Enfin, Cluzel et Philbert (2015) dans un article retraçant I'historique de l'aide humaine 
pour les élèves en situation de handicap, indiquent qu'il y a toujours eu une certaine frilosité quant à la reconnaissance de ces professionnelles, malgré une amélioration palpable ces dernières années (par ex., via la création d'une formation).

\section{Objectifs de la recherche et hypothèses}

Les différents éléments cités précédemment nous semblent traduire l'importance d'engager des travaux de recherche sur la question de la reconnaissance perçue des AESH. Ainsi, la présente recherche, bien qu'exploratoire par nature, vise à offrir de premiers éléments de compréhension à travers une comparaison de la reconnaissance perçue en fonction de la source (c.-à-d., l'institution, la hiérarchie ou les collègues enseignant·es), mais aussi l'étude d'éléments qui, dans le contexte de travail des AESH (par ex., la qualité de l'accueil reçu, la communication avec l'enseignant·e, le sentiment d'efficacité personnelle), pourraient venir influencer cette reconnaissance perçue. Si des hypothèses claires semblent difficiles à poser pour l'ensemble des liens étudiés (d'où le caractère exploratoire de cette étude), il est toutefois minimalement possible, en articulant les éléments donnés dans les parties précédentes, d'assumer que le niveau de reconnaissance perçue par les AESH de la part de l'institution devrait être plus faible que celui perçu comme provenant des directeur-trices d'école ou chef-fes d'établissement ou même que celui des enseignant·es.

Il est à noter que cette étude représente également une occasion de faire un état des lieux des conditions de travail des AESH afin d'identifier de potentiels leviers pour les améliorer. Ainsi, si nous présentons les résultats concernant la reconnaissance perçue des $\mathrm{AESH}$, nous partageons également, en détails, les éléments concernant ces conditions de travail.

\section{Méthode}

\section{Participantes}

Trois mille trois cent quatre-vingt-quatre accompagnantes d'élève en situation de handicap (AESH) se sont connectées à partir du lien envoyé et ont au moins consulté la première page du questionnaire (c.-à-d., le formulaire de présentation et de consentement). Sur ce total, $65,10 \%$ ont rempli l'intégralité du questionnaire.

L'échantillon final de l'étude comptait donc 2203 participants : 2114 femmes (96\% de l'échantillon) et 85 hommes ( $3,9 \%, 0,1 \%$ de données manquantes) avec un âge moyen de 43,12 ans $(E T=14,79)$. Sur ce total, $13,6 \%$ des participantes avaient moins d'un an d'ancienneté, 49,3\% une ancienneté comprise entre 1 et 5 ans et $37,1 \%$ une ancienneté supérieure à 5 ans. En termes de niveau de diplômes, 1,4\% des participantes n'étaient pas diplômées, 2,4\% avaient au moins le brevet des collèges, 15,7\% avaient au moins un diplôme de niveau IV (BEP/CAP), 40,4\% un baccalauréat, 17,1\% un diplôme de niveau baccalauréat +2 et $23,2 \%$ un diplôme supérieur. Ainsi, $80,7 \%$ des participantes avaient au moins un diplôme de niveau $\mathrm{V}$ (baccalauréat).

Enfin, concernant leurs conditions de travail, $60,8 \%$ des participantes exerçaient dans le premier degré, $31,4 \%$ dans le second degré et $7,8 \%$ dans un contexte mixte (premier et second degré). Une mission d'aide collective auprès des élèves en dispositif ULIS (Unités Localisées pour I'Inclusion Scolaire) était exercée par $14,4 \%$ d'entre elles alors que $85,6 \%$ exerçaient une mission d'aide 
individuelle et/ou mutualisée. Cet échantillon, en raison de sa taille et son hétérogénéité importante, nous offre une bonne représentativité.

\section{Matériel}

Dans le cadre de cette étude, il a été demandé aux participantes de compléter plusieurs mesures visant à qualifier leur expérience professionnelle, à mesurer leur perception de compétences ainsi que leur reconnaissance perçue par les différents acteur-rices de l'Éducation nationale. Le détail de ces mesures est présenté ci-dessous.

\section{Qualification de l'Expérience Professionnelle}

Nous avons cherché à établir un état des lieux le plus précis possible de la situation professionnelle des AESH à travers divers indicateurs. Notre choix s'est porté principalement sur des éléments relatifs à la mise en poste de l'AESH dans l'école ou l'établissement et à la communication entre l'AESH et l'enseignant $\cdot e$. En effet, à la lecture du livret d'accueil de l'AESH élaboré par le ministère de l'Éducation nationale et aux indices récoltés sur le terrain, une prise de poste qui permette un accompagnement qualitatif se traduit par l'accueil de I'AESH au sein de l'école ou de l'établissement par la·le supérieur.e hiérarchique et l'enseignant·e, la remise d'outils ou documents de travail nécessaires à la prise de fonction par l'équipe éducative ainsi que la présentation de l'AESH aux parents de l'élève (MENJS, 2020). Nous avons ainsi proposé des questions sur chacun de ces éléments.

Prise de poste de l'AESH. La prise de poste de l'AESH pour un nouvel accompagnement comporte l'accueil et la remise d'outils utiles à la prise de fonction. Cinq questions portaient donc sur l'accueil de I'AESH dans l'école ou l'établissement à travers la présentation des lieux, de l'équipe éducative et de l'enseignant·e de l'élève, des missions, et de l'élève (points d'appui, difficultés, objectifs et priorités concernant sa scolarisation). Par exemple, le premier item du questionnaire se présentait ainsi : "Avez-vous été accueillie dans l'école, le collège ou le lycée pour que vous soient présentés les différents lieux de vie de l'établissement ? ». En outre, dans le livret d'accueil de l'AESH, le MEN insiste sur l'indispensable entretien de présentation à la famille. Aussi la dernière question portait-elle sur celuici, avec la présence de l'enseignant ·e ou non le cas échéant : « Avez-vous rencontré lors d'un entretien de présentation les parents de l'élève que vous accompagnez? Avec l'enseignant.e?». Les participantes devaient répondre à chacune de ces questions par oui ou par non.

Les questions suivantes interrogeaient la remise d'outils ou documents de travail institutionnels tels que le projet personnalisé de scolarisation (PPS), le document de mise en œuvre du PPS (DMOPPS) et le livret d'accueil de I'AESH. Les participantes devaient, comme précédemment, répondre par oui ou par non selon si ces documents leur avaient été fournis ou pas.

Communication avec l'enseignant'e. Nous avons distingué deux types d'échanges entre I'AESH et l'enseignant $\cdot e$, centrés sur des objets différents : l'un portant sur la tâche que l'AESH a à effectuer dans l'accompagnement de l'élève dans l'accès aux activités d'apprentissage, et l'autre sur le suivi de l'élève. Les participantes devaient compléter deux énoncés relatifs aux échanges.

Le premier s'intéressait particulièrement aux échanges relatifs à la tâche : " Pour déterminer la tâche que vous avez à effectuer dans l'accompagnement de l'(les) élève(s) dans l'accès aux activités 
d'apprentissage, vous échangez avec l'enseignant $e:$ : "et était évalué sur une échelle de 1 à 6 points allant de 1 « Jamais » à 6 « Avant chaque activité d'apprentissage ».

Le second s'intéressait davantage au suivi de l'élève : "Pour assurer le suivi de(s) l'élève(s), des temps formalisés de concertation ou d'espaces de dialogue avec l'enseignant·e sont-ils prévus ? " et était également évalué sur une échelle en 6 points allant de 1 « Jamais » à 6 « Tous les jours ».

Participation aux équipes de suivi de scolarisation. À la suite, quatre énoncés portaient sur l'invitation, la participation et la préparation de l'équipe de suivi de scolarisation (ESS) avec l'enseignant·e, par exemple : «Si l'équipe de suivi de scolarisation (ESS) a eu lieu ou a été programmée, avez-vous été invitée ? » Les participantes devaient répondre par l'affirmative ou la négative.

Connaissances respectives des missions. Enfin, les participantes ont complété trois énoncés relatifs à la connaissance des missions de l'AESH par l'AESH et l'enseignant $e$, et des missions de I'enseignant $e$ dans la mise en œuvre du PPS par l'AESH. Pour chacun de ces énoncés (par ex., « Dans quelle mesure estimez-vous que l'enseignant e connaît vos missions en tant qu'AESH ? »), les participantes devaient répondre sur une échelle allant de 1 « Pas du tout » à 5 « Très bien ».

\section{Perception de Compétences}

Les participantes devaient ensuite compléter 4 énoncés extraits et adaptés de l'Échelle des Perceptions de Compétence dans les Domaines de Vie (EPCDV) de Losier, Vallerand et Blais (1993). Cette dernière mesure les perceptions de compétence entretenues par les personnes envers différents contextes de vie : les loisirs, les relations interpersonnelles, les études et envers la vie en général. Elle comprend normalement un total de 16 énoncés, soit 4 pour chacun des domaines de vie. Dans le cadre de notre étude, et pour un souci de longueur du questionnaire, nous avons fait le choix de ne retenir que 4 énoncés. Ces items ont été légèrement modifiés pour qu'ils puissent refléter la perception de compétence professionnelle (par ex., «En général, j'éprouve des difficultés à bien faire mon travail d'AESH»; "J'ai développé de très bonnes compétences comme AESH»). La consigne était: "Indiquez à quel point vous êtes en accord avec chacun des énoncés suivants ». Les participantes devaient indiquer leur degré d'accord avec chacun des énoncés sur une échelle en 5 points allant de 1 « Pas du tout d'accord » à 5 « Tout à fait d'accord ». L'analyse de fiabilité, la mesure dans laquelle cette échelle mesurait bien le construit souhaité, était relativement satisfaisante $(\alpha=, 67, M=4,03 ; E T=$ $0,61)$.

\section{Reconnaissance au travail}

Les participantes ont aussi eu à compléter l'échelle de reconnaissance au travail proposée par Fall (2015). Pour parvenir à une évaluation complète du concept de reconnaissance au travail, cet instrument de mesure prend en considération les trois dimensions de la reconnaissance au travail définies par Brun et Dugas (2005); il permet d'évaluer la reconnaissance institutionnelle ou organisationnelle, la reconnaissance verticale et la reconnaissance horizontale et donc, dans le cadre de notre étude, les perceptions que peuvent avoir les AESH de l'institution, de leurs supérieur es hiérarchiques et de leurs collègues enseignant·es. En effet, comme évoqué précédemment, le concept de reconnaissance au travail ne se limite pas uniquement à la reconnaissance du travail fournie mais 
implique aussi que cette reconnaissance soit l'œuvre d'acteur-rices bien défini·es ; dans notre étude il s'agit de l'employeur soit l'institution Éducation nationale, la directrice ou le directeur d'école ou le ou la cheffe d'établissement et les enseignant-es. Pour évaluer la sensibilité des participantes aux trois dimensions de la reconnaissance au travail, l'échelle était constituée de 13 items ; les items 1 à 4 étaient relatifs à l'employeur (par ex., "Mon employeur (l'Éducation nationale) me donne les moyens qu'il faut pour travailler efficacement »), les items 5 à 8 , au directeur ou à la directrice d'école ou la·le chefffe d'établissement (par ex., "Le(s) directeur(s) d'école ou chef(s) d'établissement dans le(s)quel(s) j'interviens fait (font) preuve de considération à mon égard (salutations, sympathie et attentions agréables). »), et les items 9 à 13 aux enseignant·es (par ex., "L'(les) enseignant(s) avec le(s)quel(s) je travaille me félicite(ent) ou me remercie(ent) pour souligner mon travail. »). Les participantes devaient alors indiquer leur degré d'accord sur une échelle en 5 points allant de 1 « Pas du tout d'accord » à 5 « Tout à fait d'accord ». II est à noter que nous avons légèrement adapté certains items et complété l'échelle en ajoutant un item concernant la reconnaissance perçue par les enseignant es. Comme pour la perception de compétences, les analyses de fiabilités étaient satisfaisantes pour la reconnaissance perçue associée à l'Éducation nationale $(\alpha=, 85, M=2,19 ; E T=0,99)$, le personnel de direction ( $\alpha$ $=, 92, M=4,05 ; E T=0,94)$ et les collègues enseignant $\cdot$ es $(\alpha=, 92, M=4,07 ; E T=0,90)$.

\section{Caractéristiques démographiques}

Enfin, afin de caractériser notre échantillon, les participantes ont été invitées à compléter neuf items relatifs au genre, à l'âge, au niveau académique, au contexte d'exercice (premier degré, second degré ou mixte), au type de mission (aide individuelle et/ou mutualisée, aide collective), à l'ancienneté, à la participation à la formation d'adaptation à l'emploi, au suivi d'une autre formation dans le domaine de l'accompagnement à la personne, ou à la projection à long terme dans le métier d'AESH. Une question ouverte clôturait le questionnaire afin de laisser la possibilité aux participantes de s'exprimer librement ou d'ajouter des précisions relatives à leur métier d'AESH'.

\section{Résultats}

\section{Description de l'expérience professionnelle des AESH}

\section{Prise de poste de l'AESH}

L'accueil de I'AESH. Les résultats concernant les quatre premiers indicateurs relatifs à la prise de poste durant l'année scolaire 2019-2020 (c.-à-d., la présentation des lieux de vie de l'école ou de l'établissement, de l'équipe éducative, des missions au regard de l'élève à accompagner ainsi que les points d'appui et difficultés de l'élève) traduisent un retour positif pour plus de $60 \%$ des AESH.

Néanmoins, seules $32,1 \%$ d'entre elles indiquent avoir rencontré les parents de l'élève alors que cela est prescrit (circulaire ${ }^{\circ} 2019-088$ du 5-6-2019). Le détail des réponses pour chaque indicateur est donné dans le tableau ci-dessous.

\footnotetext{
łPour des questions de lisibilité, l'intégralité du questionnaire n’est pas présentée ici car toutes les variables n'ont pas fait l'objet d'une analyse. Néanmoins, l'ensemble du matériel et données sont disponibles en suivant le lien suivant : https://osf.io/dgp7n/
} 



\section{Tableau 1}

L'accueil des AESH

Indicateurs d'accueil de l'AESH

Présentation des lieux de vie

Oui

$68,50 \%$

Non

Présentation de l'équipe éducative

$65,10 \%$

$64,60 \%$

$31,50 \%$

Présentation des missions

$61,30 \%$

$34,90 \%$

Présentation de l'élève

$32,10 \%$

$35,40 \%$

$38,70 \%$

Présentation des parents de l'élève

La remise d'outils à l'AESH. Des outils institutionnels tels que le Projet Personnalisé de Scolarisation (PPS), le document de mise en œuvre du PPS (DMOPPS), le livret d'accueil de l'AESH ou d'autres documents de travail devraient être remis à l'AESH pour l'aider dans sa prise de fonction. Les résultats font apparaître que, selon les dires des participantes, ces documents ne sont pas systématiquement distribués (moins de $50 \%$ des AESH déclarent avoir reçu chaque outil). Le détail par outil est présenté dans le tableau ci-dessous.

\section{Tableau 2}

Remise d'outils aux AESH

\begin{tabular}{lcc}
\multicolumn{1}{c}{ Outils ou documents de travail } & Oui & Non \\
\hline PPS & $46,40 \%$ & $53,60 \%$ \\
DMOPPS & $31,90 \%$ & $68,10 \%$ \\
Livret d'accueil de l'AESH & $46,30 \%$ & $53,70 \%$ \\
Autre document & $21,10 \%$ & $78,90 \%$ \\
\hline
\end{tabular}

En outre, il est à noter que concernant les $21,1 \%$ des participantes qui ont reçu un autre document ou outil de travail, il s'agit essentiellement du guide d'évaluation des besoins de compensation en matière de scolarisation (GEVA-Sco) et/ou du projet d'accueil individualisé (PAI) pour les élèves avec troubles de la santé.

\section{Communication avec l'enseignant $\cdot e$}

Nous avons distingué deux types d'échanges entre l'AESH et l'enseignant $\cdot e$ : des échanges portant sur la tâche que l'AESH doit effectuer dans l'accompagnement de l'élève dans l'accès aux activités d'apprentissage, et des échanges centrés sur le suivi de l'élève dans sa scolarisation.

Concernant la tâche à effectuer. Les résultats concernant les échanges relatifs à la tâche à effectuer par l'AESH sont détaillés dans le tableau ci-dessous.

Ainsi, il apparaît notamment que 11,94\% des participantes déclarent ne jamais échanger avec l'enseignant $\cdot e$ pour déterminer la tâche qu'elles ont à effectuer dans l'accompagnement de l'élève dans l'accès aux activités d'apprentissage. Toutefois, il semble qu'environ la moitié des AESH qui ont participé à l'étude échangent avec l'enseignant·e avant ou pendant l'activité d'apprentissage (c.-à-d., $51,74 \%)$. 


\section{Tableau 3}

Fréquence relative aux échanges concernant la tâche

Pourcentage des participantes

\begin{tabular}{lc}
\hline Avant chaque activité d'apprentissage & $27,14 \%$ \\
Au cours de chaque activité d'apprentissage & $24,60 \%$ \\
Deux fois par jour & $7,81 \%$ \\
Une seule fois par jour & $13,25 \%$ \\
Une fois par semaine & $15,25 \%$ \\
Jamais & $11,94 \%$ \\
\hline
\end{tabular}

Concernant le suivi des élèves. $39,85 \%$ des AESH ont indiqué n'avoir aucun temps formalisé de concertation ou d'espaces de dialogue avec l'enseignant $\cdot e$ au sujet du suivi de l'élève. Le détail des résultats est présenté dans le tableau ci-dessous.

\section{Tableau 4}

Fréquence relative aux échanges concernant le suivi de l'élève

\begin{tabular}{lc}
\hline Tous les jours & $31,59 \%$ \\
Une fois par semaine & $17,16 \%$ \\
Une fois par mois & $3,50 \%$ \\
Une fois par trimestre & $4,72 \%$ \\
Une fois par an & $3,18 \%$ \\
Jamais & $39,85 \%$ \\
\hline
\end{tabular}

Équipes de suivi de scolarisation (ESS). Dans le cadre des ESS, 90,2\% des participantes ont répondu avoir été invitées et, lorsque l'ESS a eu lieu, 90,6\% des AESH y ont participé. Parmi celles qui ont participé à l'ESS, 57,4\% l'ont préparée avec l'enseignant·e (42,6\% sans l'enseignant·e).

Si ces éléments n'ont que vocation à décrire le contexte de travail des AESH, ils seront néanmoins supports dans l'analyse des déterminants de la reconnaissance perçue et seront également évoqués en discussion.

\section{Reconnaissance perçue des AESH}

\section{Reconnaissance perçue en fonction de la source}

Une première analyse de variance (ANOVA) à mesures répétées sur la reconnaissance perçue incluant la source comme facteur intra-sujet à trois niveaux a été réalisée (c.-à-d., soit l'Éducation nationale, la·le directeur-rice ou la·le chef·fe d'établissement et l'enseignant·e). Celle-ci indique que le niveau de reconnaissance varie significativement selon la source, $F(2,4404)=4085,35, p<.001, \eta_{p}^{2}$ $=, 65$. Plus précisément, et comme l'illustre le graphique ci-dessous, les analyses de comparaisons a posteriori avec correction de Bonferonni indiquent que les participantes perçoivent beaucoup moins de reconnaissance de la part de l'Éducation nationale $(M=2,19, E S=.02)$ par comparaison aux directeur.rices et chef·fes d'établissement $(M=4,05, E S=, 02, p<.001)$ et aux enseignant'es ( $M$ $=4,07, E S=.02, p<.001$ ) ; le niveau de reconnaissance perçue ne varie pas entre ces deux dernières sources $(p=1.00)$. Autrement dit, confirmant les impressions récoltées sur le terrain, les AESH interrogées indiquent se sentir beaucoup moins reconnues par l'institution que par les acteur rices avec lesquel·les elles interagissent au quotidien. 


\section{Figure 1}

Reconnaissance perçue des AESH selon les différentes sources. Les barres d'erreurs représentent les intervalles de confiance.

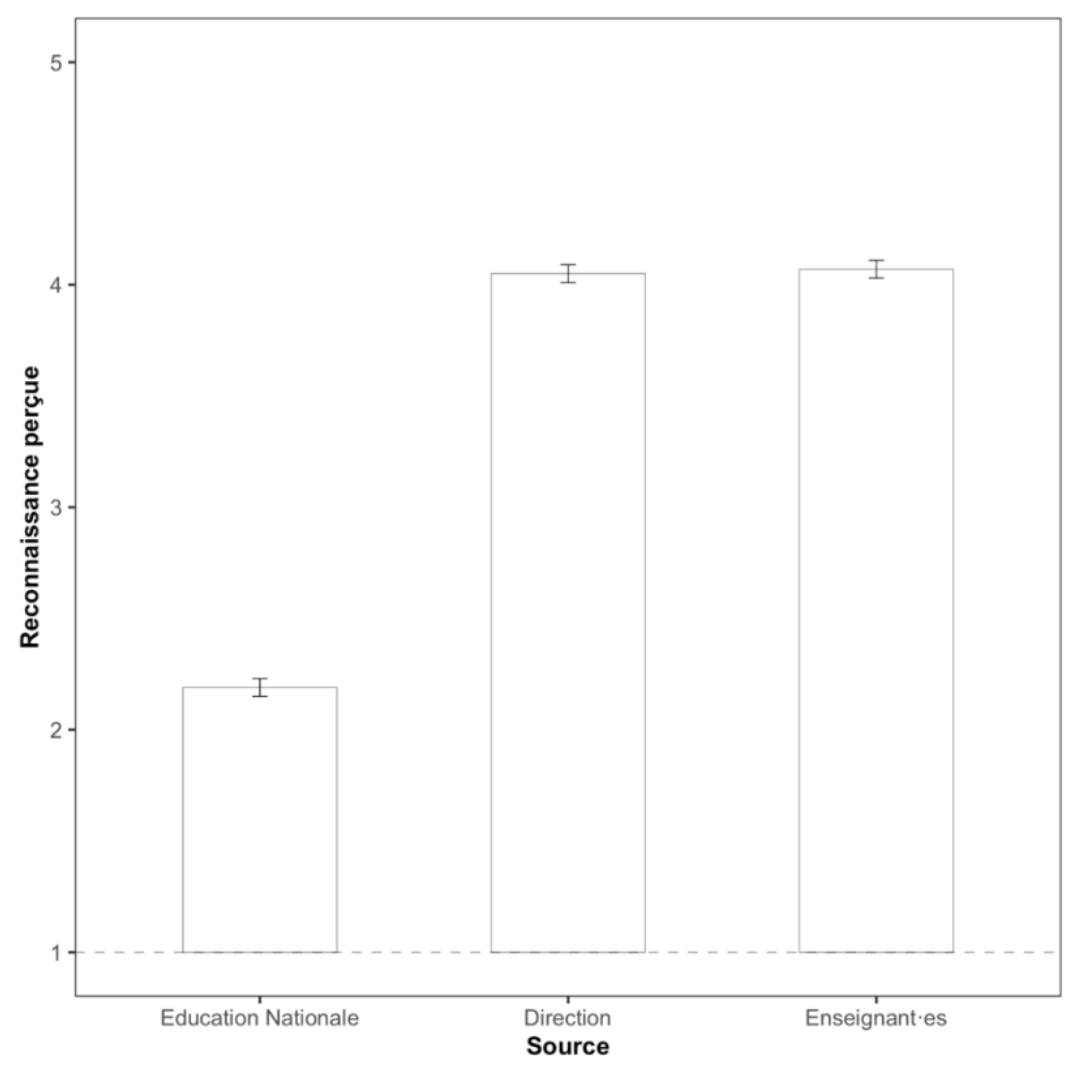

\section{Liens entre contexte professionnel et reconnaissance perçue des AESH}

Dans un second temps d'analyse, nous avons examiné les déterminants de cette reconnaissance perçue à l'aide des indicateurs présentés précédemment. Ainsi, les trois scores de reconnaissance perçue ont été régressés sur des modèles incluant plusieurs facteurs que sont : l'accueil (l'ensemble des oui aux questions dédiées était additionné pour former un score compris entre 0 et $5, M=2,92 ; E T=1,59$ ), la transmission d'outils (l'ensemble des oui aux questions dédiées était additionné pour former un score compris entre 0 et $4, M=1,46 ; E T=1,27$ ), la communication concernant la tâche à effectuer $(M=3,99 ; E T=1,77)$, le suivi de l'élève $(M=3,50 ; E T=2,22)$, la participation aux ESS (l'ensemble des oui aux questions dédiées était additionné pour former un score compris entre 0 et $4, M=3,06 ; E T=1,18)$, la connaissance perçue des missions de l'enseignant $e(M$ $=3,28 ; E T=1,17)$, la connaissance des missions de l'AESH $(M=4,12 ; E T=0,78)$, la connaissance perçue des missions de l'AESH par l'enseignant e $(M=3,44 ; E T=1,09)$, le sentiment de compétence, l'expérience professionnelle dans le métier (codée +1 pour une expérience de moins de un an, +2 pour une expérience comprise entre 2 et 5 ans et +3 pour une expérience supérieure à 5 ans), le contexte de travail (codé +1 pour le premier degré, +2 pour le second degré et +3 pour un contexte mixte) et des caractéristiques démographiques (c.-à-d., âge, genre, niveau de diplôme). 
Plutôt qu'une présentation très détaillée de l'ensemble des résultats issus de ces trois analyses de régression (pour le détail, voir le tableau 5 ci-dessous), nous proposons de mettre en lumière les points les plus marquants.

Tout d'abord, il existe des convergences entre les trois analyses. Ainsi, il semble que plus les AESH reçoivent un bon accueil, échangent concernant la tâche à effectuer, suivent l'élève avec l'enseignant·e, pensent que celui-ci ou celle-ci connaît leurs missions, et se sentent compétentes dans leur emploi, plus elles indiquent ressentir de la reconnaissance de la part de l'institution, la hiérarchie ou les collègues enseignant·es. Dit autrement, pour que les AESH se sentent reconnues dans leur travail, quelle que soit la source de cette reconnaissance, il semble indispensable de favoriser l'accueil sur le lieu de travail ou encore les échanges réguliers (à propos de la tâche ou l'élève) avec un·e enseignant $\cdot e$ dont elles pensent qu'il $\cdot$ elle connaît bien leurs missions. Si les autres paramètres apparaissent comme liés à la reconnaissance perçue, cela semble varier selon la source de celle-ci.

Ainsi, et cela est surprenant, si la participation aux ESS est associée à une meilleure reconnaissance perçue par la direction ou les enseignant ·es, c'est l'inverse qui se produit pour la reconnaissance institutionnelle, comme si cela représentait un frein. Autre exemple, l'expérience de I'AESH est associée à la reconnaissance perçue de l'institution et de la direction mais pas des enseignant·es (c.-à-d., si les AESH les moins expérimentées se sentent davantage reconnues par l'institution et la direction que leurs collègues plus expérimentées, cette différence n'apparaît plus quand la source est l'enseignant $\cdot e)$. Un résultat similaire concerne le niveau de diplôme, avec les AESH les moins diplômées qui se sentent davantage reconnues par l'institution et la direction que leurs collègues plus diplômées, une différence qui n'apparait pas pour la reconnaissance perçue associée à l'enseignant·e.

Enfin, certains paramètres semblent avoir une influence très spécifique et ne sont associés qu'à une seule forme de reconnaissance perçue. C'est notamment le cas de l'âge, du genre, du contexte professionnel, de la participation à la formation d'adaptation à l'emploi ou encore de la transmission d'outils qui ne sont associés qu'à la seule reconnaissance perçue de l'institution. Plus précisément, les AESH les plus jeunes, du premier degré, hommes, qui n'ont pas fait la formation d'adaptation et qui ont le moins reçu d'outils institutionnels sont ceux qui se sentent le moins reconnus par l'institution.

Il est à noter, de manière consistante, que les connaissances que les $\mathrm{AESH}$ possèdent à propos de leurs missions ou celles de l'enseignant·e n'influencent pas leur reconnaissance perçue, quelle que soit la source. 


\section{Tableau 5}

Synthèse des analyses de régression concernant les déterminants de la reconnaissance perçue des AESH selon la source de celle-ci.

\begin{tabular}{|c|c|c|c|}
\hline & Éducation nationale & Direction & Enseignant·es \\
\hline Accueil de l'AESH & 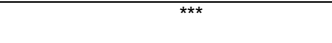 & $\begin{array}{c}* \star \\
\end{array}$ & \\
\hline Transmission des outils institutionnels & *** & ns & ns \\
\hline $\begin{array}{l}\text { Communication à propos de l'activité à faire } \\
\text { avec l'élève }\end{array}$ & $\star \star * *$ & $* * *$ & $* * *$ \\
\hline Suivi de l'élève & *** & *** & *** \\
\hline Participation aux ESS & 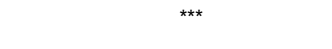 & *** & *** \\
\hline $\begin{array}{l}\text { Connaissances des missions de } \\
\text { l'enseignant } \cdot e\end{array}$ & ns & ns & ns \\
\hline Connaissances des missions de l'AESH & ns & ns & ns \\
\hline $\begin{array}{l}\text { Connaissances des missions de l'AESH par } \\
\text { l'enseignant·e }\end{array}$ & *** & 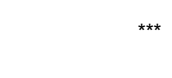 & $* * *$ \\
\hline Sentiment de compétence & *** & *** & *** \\
\hline Age de l'AESH & ** & ns & ns \\
\hline Niveau de diplôme & *** & *** & $\mathrm{ns}$ \\
\hline Contexte de travail & *** & ns & ns \\
\hline Expérience professionnelle de l'AESH & *** & *** & ns \\
\hline $\begin{array}{l}\text { Participation à la formation d'adaptation à } \\
\text { l'emploi }\end{array}$ & ** & ns & ns \\
\hline Genre & * & ns & ns \\
\hline
\end{tabular}

Note. ${ }^{\text {ns }} p>.05 ;{ }^{*} p<.05 ;{ }^{* *} p<.01 ;{ }^{* * *} p<.001$

\section{Discussion}

Les AESH ont un rôle particulièrement important dans la mise en place de l'école inclusive mais souffrent néanmoins d'un manque de reconnaissance (SNALC, 2017). S'inscrivant en partie dans la perspective de travaux menés en Sciences de gestion sur cette problématique (Brun \& Dugas, 2005 ; Fall, 2015), l'objectif du présent travail était d'étudier la question de cette reconnaissance de manière quantitative afin d'identifier des éléments pouvant l'influencer (par ex., la source, les conditions de travail). Une étude exploratoire auprès d'un large échantillon d'AESH a donc été menée afin d'apporter des éléments de réponse à cette question mais aussi afin de fournir des éléments descriptifs sur le contexte de travail de ces professionnelles.

De manière synthétique, les résultats de l'étude confirment, sans équivoque, que les AESH perçoivent un fort déficit de reconnaissance de la part de l'Éducation nationale (notamment par contraste avec celle perçue comme venant des personnels de direction ou des enseignant·es) et que celui-ci serait partiellement imputable à leurs conditions de travail. En effet, il apparaît dans les résultats que la réalisation systématique de l'accueil des AESH lors de la prise de poste - encore trop faible, seules $32,1 \%$ rencontrent les parents de l'élève qu'elles accompagnent malgré le caractère prescrit de cette rencontre -, la transmission systématique des outils institutionnels - elle aussi trop faible, seules $46,40 \%$ des AESH auraient reçu le PPS -, ou encore le simple fait d'avoir bénéficié de la formation d'adaptation à l'emploi constitueraient autant de pistes permettant d'améliorer le sentiment de reconnaissance de ces personnels et ainsi leur offrir un cadre probablement plus propice à la mise en œuvre d'un accompagnement plus qualitatif.

De même, il apparaît que les enseignant·es pourraient contribuer significativement à l'amélioration de la reconnaissance des AESH. Ainsi, en témoignant simplement aux AESH d'une 
connaissance appropriée de leurs missions et en échangeant régulièrement avec elles sur les activités à mener en classe ou le suivi de l'élève, cela pourrait déjà permettre d'améliorer significativement la reconnaissance qu'elles perçoivent. En effet, le fait qu'environ $26 \%$ des AESH de notre échantillon n'échangent pas au moins une fois par jour sur les activités à mener en classe ou que près de $40 \%$ d'entre elles n'aient jamais de temps de concertation formalisé avec l'enseignant·e sur l'élève traduit, semble-t-il, des dysfonctionnements entrainant probablement des difficultés dans la mise en œuvre de la mission d'accompagnement des élèves en situation de handicap.

Ce dernier point nous semble renforcer significativement l'idée que pour collaborer efficacement, il faut offrir des espaces aux individus pour qu'ils puissent apprendre à travailler ensemble (Tomazet \& Mérini, 2014). Ainsi, si cela peut passer par de la formation aux enseignant·es quant aux missions de l'AESH et son rôle dans la classe via, peut-être, des formations croisées $\mathrm{AESH}$-enseignant·es, cela passe probablement aussi par la mise à disposition d'outils de travail collaboratif. Cochetel (2019) en recense un certain nombre qui pourraient être facilement mis en place. Par exemple, afin d'améliorer le suivi de l'élève, pourrait être proposé de manière systématique un cahier de liaison permettant aux membres du binôme de consigner les éléments jugés utiles à l'évolution du projet de scolarisation de l'élève. Autre exemple, l'utilisation systématique d'une grille d'observation et d'évaluation par l'AESH portant sur l'autonomie de l'élève accompagné dans la vie quotidienne, les apprentissages ou la vie au sein de l'école offrirait à l'AESH un support pour soutenir sa position lors des temps de suivi ou réunions concernant l'élève. Enfin, le livret de parcours inclusif évoqué lors du dernier comité national de suivi de l'école inclusive du 9 novembre 2020 pourrait également constituer à l'avenir un outil de communication et d'échanges facilitant le suivi du parcours de scolarisation de l'élève ainsi que la prise de poste de l'AESH.

Ainsi si ce travail apporte des éléments de réflexion, il n'est toutefois pas sans certaines limites qu'il convient de considérer. Premièrement, les données présentées ici ne sont qu'auto-rapportées. Les biais associés à ce type de données sont connus (par ex., le biais de désirabilité sociale, Paulhus, 1991) et nous incitent donc à la prudence quant aux conclusions à formuler. Un travail complémentaire, impliquant une méthode qualitative, permettrait très probablement d'affiner et d'aller plus loin dans la compréhension de l'origine de ce manque de reconnaissance. Deuxièmement, notre échantillon, bien que large, pourrait ne pas être pour autant très représentatif de l'ensemble des AESH. En effet, si nous avons essayé de diffuser largement le questionnaire, les réseaux l'ayant le plus relayé sont ceux qui sont particulièrement engagés pour l'amélioration des conditions de travail des AESH. II n'est ainsi pas à exclure un biais de sélection dans notre échantillon avec un taux de réponse particulièrement important parmi les AESH les plus engagées sur cette question-là. Enfin, le caractère exploratoire de l'étude appelle à une nécessaire réplication de ces travaux afin de pouvoir consolider les résultats obtenus ici (Makel \& Plucker, 2014).

Au-delà de ces limites, et au regard des conséquences que la reconnaissance professionnelle peut avoir sur la motivation ou encore le sentiment de bien-être des personnels (Fall, 2015), il nous semble que les résultats présentés ici ne sont pas neutres et doivent initier une réflexion vive autour des conditions de travail des AESH. Si les annonces de la rentrée 2020 du ministère de l'Éducation 
nationale (c'est-à-dire, création d'un guide national des $\mathrm{AESH}$, réaffirmation de l'importance des échanges avec la famille, déploiement des PIAL renforcés et création de postes d'AESH référentes) incitent à une forme d'optimisme, beaucoup reste à faire pour permettre à ces personnels d'être pleinement reconnus dans leur travail et ainsi probablement offrir aux élèves en situation de handicap de meilleures conditions pour la poursuite de leur scolarité.

\section{Bibliographie}

Anderson, C., Hildreth, J. A. D., \& Howland, L. (2015). Is the desire for status a fundamental human motive? A review of the empirical literature. Psychological Bulletin, 141(3), 574-601. https://doi.org/10.1037/a0038781

Anonyme. (2017). AVS, un métier à tisser ? AVS c'est un métier! Vie Sociale et Traitements, Revue des CEMEA, 135(3), 61-65. https://doi.org/10.3917/vst.135.0061

Brillet, F., \& Capdevielle, M. (2017). La fonction publique territoriale face aux attentes de ses agents en matière de reconnaissance. Recherches en Sciences de Gestion, 120(3), 143-166. https://doi.org/10.3917/resg.120.0143

Brun, J. \& Dugas, N. (2005). La reconnaissance au travail : analyse d'un concept riche de sens. Gestion, 30(2), 79-88. https://doi.org/10.3917/riges.302.0079

Cluzel, S., \& Philbert, M. C. (2015). L'accompagnement des jeunes en situation de handicap: l'ambition d'un métier. Contraste, 42(2), 205-222. https://doi.org/10.3917/cont.042.0205

Cochetel, G. (2019). AESH et enseignant, collaborer dans une école inclusive. Réseau Canopé.

Fall, A. (2015). Reconnaissance au travail : Validation d'une échelle de mesure dans le contexte des entreprises. European Review of Applied Psychology, 65(4), 189-203.

https://doi.org/10.1016/j.erap.2015.07.001

Komites, P. (2013). Rapport du groupe de travail «Professionnaliser les accompagnants pour la réussite des enfants et adolescents en situation de handicap» consulté le 12 août 2020 à l'adresse https://solidarites-sante.gouv.fr/IMG/pdf/rapport-pkomites-2013.pdf

Losier, G. F., Vallerand, R. J., \& Blais, M. R. (1993). Construction et validation de l'Échelle des Perceptions de Compétence dans les Domaines de Vie (EPCDV). Science et comportement, 23(1), 1-16.

Maguet, U., \& Panissal, N. (2019). AESH au service d'une école inclusive et bienveillante: quelles compétences éthiques?. Pensee plurielle, 49(1), 49-59. https://doi.org/10.3917/pp.049.0049

Makel, M. C., \& Plucker, J. A. (2014). Facts are more important than novelty: Replication in the education sciences. Educational Researcher, 43(6), 304-316. https://doi.org/10.3102/0013189X14545513

Paulhus, D. L. (1991). Measurement and control of response bias. In J. P. Robinson, P. R. Shaver, \& L. S. Wrightsman (Eds.), Measures of social psychological attitudes, Vol. 1. Measures of personality and social psychological attitudes (p. 17-59). Academic Press. https://doi.org/10.1016/B978-0-12-590241$\underline{0.50006-X}$

Philip, C., \& Philbert, M.-C. (2009). Auxiliaire de vie scolaire, un nouveau métier ? La nouvelle revue de l'adaptation et de la scolarisation, 45, 1-238. https://www.inshea.fr/fr/content/auxiliaire-de-viescolaire-un-nouveau-métier

Renger, D., Miché, M., \& Casini, A. (2020). Professional recognition at work: The protective role of esteem, respect, and care for burnout among employees. Journal of Occupational and Environmental Medicine, 62(3), 202-209. https://doi.org/10.1097/JOM.0000000000001782 
Thomazet, S., et Mérini, C. (2014). Le travail collectif, outil d'une école inclusive ? Questions vives recherches en éducation, 21, 1-18. https://doi.org/10.4000/questionsvives. 1509

Thomazet, S., \& Mérini, C. (2015). L'école inclusive comme objet frontière. La nouvelle revue de l'adaptation et de la scolarisation, 70-71(2/3), 137-148. https://doi.org/10.3917/nras.070.0137

Toullec-Théry, M. (2019). D’une recherche sur le travail conjoint AESH-enseignants vers la mise en œuvre d'une ingénierie coopérative: un moyen pour renouveler les pratiques?. La nouvelle revue-Education et societe inclusives, 85(1), 19-36. https://doi.org/10.3917/nresi.085.0019 